\title{
Impact of Rotavirus Vaccination in Severe Rotavirus Gastroenteritis Outpatient Visits at Three Pediatric Primary Care Clinics in Shibata City, Niigata Prefecture, Japan
}

\author{
Tomohiro Oishi ${ }^{*}$, Shinya Tsukano², Tokushi Nakano³, Shoji Sudo4, Hiroaki Kuwajima5, \\ for the Shibata RVGE Study Group \\ ${ }^{1}$ Department of Pediatrics, Niigata University Medical and Dental Hospital, Niigata City, Niigata, Japan \\ ${ }^{2}$ Pediatric Department, Niigata Prefectural Shibata Hospital, Shibata City, Niigata, Japan \\ ${ }^{3}$ Nakano Children's Clinic, Shibata City, Niigata, Japan \\ ${ }^{4}$ Sudo Pediatric Clinic, Shibata City, Niigata, Japan \\ ${ }^{5}$ Pediatric Department, Kuwajima Clinic, Shibata City, Niigata, Japan \\ Email:
}

Received 16 September 2014; revised 14 October 2014; accepted 10 November 2014

Copyright (C) 2014 by authors and Scientific Research Publishing Inc.

This work is licensed under the Creative Commons Attribution International License (CC BY).

http://creativecommons.org/licenses/by/4.0/

c) (i) Open Access

\begin{abstract}
The impact of rotavirus (RV) vaccination in reducing severe rotavirus gastroenteritis (RVGE) in outpatient settings was prospectively surveyed in three pediatric clinics in Shibata City. In children younger than 3 years of age, the occurrence of severe RVGE among all acute gastroenteritis (AGE) was found to be significantly lower in three seasons after introduction of RV vaccines, compared to that in 2011, before introduction of RV vaccines. The incidence rates of severe RVGE among children younger than 3 years of age were found to be reduced by $71.2 \%, 47.7 \%$, and $81.1 \%$ for 2012,2013 , and 2014, respectively, compared to that in 2011 . These results suggest that the $R V$ vaccination is effective for the prevention of severe RVGE in Japanese voluntary RV vaccination settings with estimated coverage rates of $32.5 \%, 40.5 \%$ and $47.1 \%$ for 2012,2013 and 2014 , respectively. It is expected that the reducing effect on severe RVGE would be persistently established by increasing the vaccine coverage rates.
\end{abstract}

\section{Keywords}

Impact of Vaccination, Rotavirus Gastroenteritis, Rotavirus Vaccine, Prospective Observation, Outpatient Settings

\footnotetext{
${ }^{*}$ Corresponding author.
}

How to cite this paper: Oishi, T., et al., for the Shibata RVGE Study Group (2014) Impact of Rotavirus Vaccination in Severe Rotavirus Gastroenteritis Outpatient Visits at Three Pediatric Primary Care Clinics in Shibata City, Niigata Prefecture, Japan. Open Journal of Pediatrics, 4, 291-299. http://dx.doi.org/10.4236/ojped.2014.44040 


\section{Introduction}

Rotavirus (RV) infection is the most common cause of severe diarrhea worldwide [1]. Patients with rotavirus gastroenteritis (RVGE) may develop severe dehydration, which can be life-threatening if the body fluid imbalances are not appropriately corrected. Although fatal cases are rare in Japan, it has been reported that approximately 790,000 children younger than 6 years of age visit hospitals/clinics as outpatients due to RVGE [2], and approximately 78,000 children younger than 5 years of age are hospitalized annually [3].

Currently, two oral RV vaccines, Rotarix ${ }^{\mathrm{TM}}$ (GlaxoSmithKline Biologicals, Rixensart, Belgium) and RotaTeq ${ }^{\mathrm{TM}}$ (Merck \& Co., Inc., Whitehouse Station, NJ, USA), for prevention of RVGE are approved in more than 100 countries and are incorporated into national immunization programs in more than 50 countries by April 2014 [4]. In some of the countries in which these RV vaccines have been introduced, the number of hospitalizations due to RVGE has dramatically decreased by the direct and indirect effects resulting from the vaccinations [4]-[6]. Since reviewing recent evidence on efficacy and safety of RV vaccines, the WHO has recommended to use RV vaccines in all national immunization programs [7]. In Japan, Rotarix ${ }^{\mathrm{TM}}$ and RotaTeq ${ }^{\mathrm{TM}}$ have been on the market since November 2011 and July 2012, respectively. Although RV vaccination had not been adopted into the national immunization program as of 2014 , an estimation of $45 \%$ uptake with a wide range of variation throughout Japan was reported in 2013 [8].

To assess the impact of the RV vaccination, an observational study design examining changes in disease burden overtime, including seasons before and after the introduction of RV vaccines, is commonly used [9]. Many of the studies reporting RV vaccine efficacy in the real world [4]-[6], indicate the impact of RV vaccination based on the data from a national database or a large insurance database. In Japan, we do not have national statistics of RV-related diseases and it is difficult to show the nationwide vaccine impact. The number of infectious gastroenteritis cases including RVGE has been collected weekly from sentinel pediatric clinics since the years before vaccine introduction, but the actual number of RVGE cases cannot be specified. For RVGE hospitalizations, the number of patients started to be collected from sentinel hospitals just after introduction of vaccines.

This study was planned to prospectively assess the impact of RV vaccines in a small city in Japan. The pragmatic design to evaluate the vaccine impact in outpatient settings using the number of severe RVGE patients as an indicator was developed by referring to some hospital-based [10]-[12] and primary practice-based [13] impact studies conducted in other countries. We previously reported the early impact by comparing the 2011 and 2012 RVGE epidemic seasons [14]. Here we report the impact observed in three RV epidemic seasons after RV vaccine introduction in 2011.

\section{Method}

This prospective observational study was conducted at three primary-care pediatric clinics in Shibata City, Niigata Prefecture, Japan. Shibata City is a small city with a stable population of approximately 100,000 inhabitants, and around 770 babies are born each year. In the city there are three primary-care pediatric clinics and one hospital with a pediatric ward, the Niigata Prefectural Shibata Hospital (Shibata Hospital). The three pediatric clinics serve pediatric primary medicine for people living in Shibata City and surrounding areas. The Shibata Hospital is the central facility in Kaetsu medical area, one of the seven secondary medical areas in Niigata Prefecture. We assumed that almost all children living in Shibata City would visit one of the three pediatric clinics when they have an illness such as acute gastroenteritis (AGE) and would be presented to the Shibata Hospital when they need a hospitalization care.

Children younger than 5 years of age (or 3 years of age for 2011) who visited a study site from February 2011 to May 2014 due to AGE were included. We recorded date of visit, date of birth, sex, and living area of all AGE patients with their symptoms (presence or absence of diarrhea and vomiting) and whether they required intravenous rehydration to correct dehydration. AGE was defined as occurrence of gastroenteritis symptoms for less than 14 days. Patients who required intravenous rehydration were defined as having severe AGE and were tested for fecal RV antigen. Rapid fecal rotavirus tests were performed using ImmunoCard ${ }^{\mathrm{TM}}$ ST Rotavirus (Fujirebio Inc., Tokyo, Japan) after oral informed consent was obtained from the parents/guardians of the pediatric patients. Severe AGE patients with positive RV antigen test results were defined as having severe RVGE and detailed information regarding their symptoms was collected to calculate severity score using modified Vesikari's scale [14] [15]. 
Clinical research records of AGE patients who lived in Shibata City were analyzed. Comparison of occurrence and incidence of RVGE before and after RV vaccine introduction was performed for only patients younger than 3 years of age during each epidemic season, because we collected the information on AGE patients younger than 3 years of age and only for epidemic months in 2011, the season before RV vaccine introduction.

A Chi-square test was used to calculate the statistical significance of differences between distributions of categorical variables, and Student's $t$-test was used to compare means of numerical variables. The incidences and 95\% confidence intervals of outpatient severe RVGE, severe GE, and all acute GE were calculated based on the numbers of patients who visited the three clinics during each observation period and the number of children under 3 years of age who lived in Shibata City. These incidences were compared between 2011 and the other years using Poisson regression analysis. Microsoft Excel version 14 was used for statistical analyses.

Genotyping of rotavirus was performed when a sufficient amount of sample was obtained from a patient. Analyses were performed using reverse transcription-polymerase chain reaction at Niigata Prefectural Institute of Public Health and Environmental Sciences. All RV vaccination records in the three pediatric clinics and the Shibata Hospital were also retrospectively collected, to estimate vaccine coverage rates. The number of patients with AGE and RVGE hospitalized in the Shibata Hospital from 2008 to 2014 was retrospectively collected for reference.

This study was conducted in compliance with Ethical Guidelines for Epidemiological Research (June 17, 2002 [Partial revision: December 1, 2008] The Ministry of Education, Culture, Sports, Science and Technology; Ministry of Health, Labour and Welfare) based on the spirit of the Declaration of Helsinki - Ethical Principles for Medical Research Involving Human Subjects. Prior to conducting the study, the Ethical Review Committees of the Niigata Prefectural Shibata Hospital reviewed both ethical and scientific aspects of the study protocol (e.g., study design, population, time period, etc.) and approved the proposed study conduct.

\section{Results}

\subsection{Number of Episodes Observed and Analyzed}

In total, 3778 AGE episodes were observed in children younger than 5 years of age who lived in Shibata City at the three pediatric clinics during the study period, from February 14, 2011 to May 31, 2014. Of which, 2203 were observed during the epidemic months, from February to May. Of the total AGE episodes, 2589 occurred in children younger than 3 years of age during the extended period and 1473 during the epidemic months. The number of AGE episodes of each year is summarized in Table 1. We analyzed 1473 AGE episodes in children younger than 3 years of age who visited a pediatric clinic during the epidemic months.

Yearly numbers analyzed are shown in Table 2. Of 1473 AGE episodes, 147 (10.0\%) were defined as severe AGE because intravenous rehydration was applied for them. Of 147 patients with severe AGE, 137 (93.2\%) were tested for fecal RV antigen and 101 (73.7\%) were confirmed as severe RVGE. For comparison of the occurrence and incidence of severe RVGE before and after RV vaccine introduction, we included 10 episodes of

\begin{tabular}{|c|c|c|c|c|}
\hline Year & Observation period & $<3$ years of age & 3 - 4 years of age & Total $(<5$ years of age \\
\hline \multirow{2}{*}{2011} & Epidemic months ${ }^{*}$ & 397 & - & $397^{* * *}$ \\
\hline & Extended period $^{* *}$ & 542 & - & $542^{* * *}$ \\
\hline \multirow[t]{2}{*}{2012} & Epidemic months ${ }^{*}$ & 355 & 214 & 569 \\
\hline & Extended period $^{* *}$ & 857 & 407 & 1264 \\
\hline \multirow[t]{2}{*}{2013} & Epidemic months ${ }^{*}$ & 398 & 242 & 640 \\
\hline & Extended period $^{* *}$ & 783 & 452 & 1235 \\
\hline \multirow[t]{2}{*}{2014} & Epidemic months ${ }^{*}$ & 323 & 274 & 597 \\
\hline & Extended period $^{* *}$ & 407 & 330 & 737 \\
\hline \multirow[t]{2}{*}{ Total } & Epidemic months & 1473 & 730 & 2203 \\
\hline & Extended period & 2589 & 1189 & 3778 \\
\hline
\end{tabular}

*Epidemic months: February 14 to May 31 for 2011, and February 1 to May 31 for 2012, 2013, 2014; ${ }^{* *}$ Extended period: February 14 to May 31 and October 1 to December 31 for 2011, January 1 to December 31 for 2012 and 2013, January 1 to May 31 for 2014 ; ${ }^{* * *}<3$ years of age. 
severe AGE not tested for fecal RV antigen in addition to 101 episodes of test-confirmed severe RVGE, to avoid overestimation of the vaccine impact. Monthly numbers of AGE, severe AGE, and severe RVGE are shown in Figure 1. The peak month of severe RVGE was April in each year.

\subsection{Changes in Occurrence of Severe RVGE}

The occurrences of severe RVGE among severe AGE and all AGE, and severe AGE among all AGE are summarized in Table 3. The occurrences of severe RVGE among all AGE and severe AGE among all AGE in 2012,

Table 2. Number of episodes analyzed ( $<3$ years of age, February to May).

\begin{tabular}{|c|c|c|c|c|c|}
\hline Type of episodes & 2011 & 2012 & 2013 & 2014 & Total \\
\hline AGE & 397 & 355 & 398 & 323 & 1473 \\
\hline Severe AGE & 62 & 31 & 41 & 13 & 147 \\
\hline Severe AGE tested for fecal RV antigen & 62 & 28 & 39 & 8 & 137 \\
\hline Severe RVGE confirmed & 52 & 14 & 29 & 6 & 101 \\
\hline Severe RVGE (including episodes not tested) & 52 & 17 & 31 & 11 & 111 \\
\hline
\end{tabular}

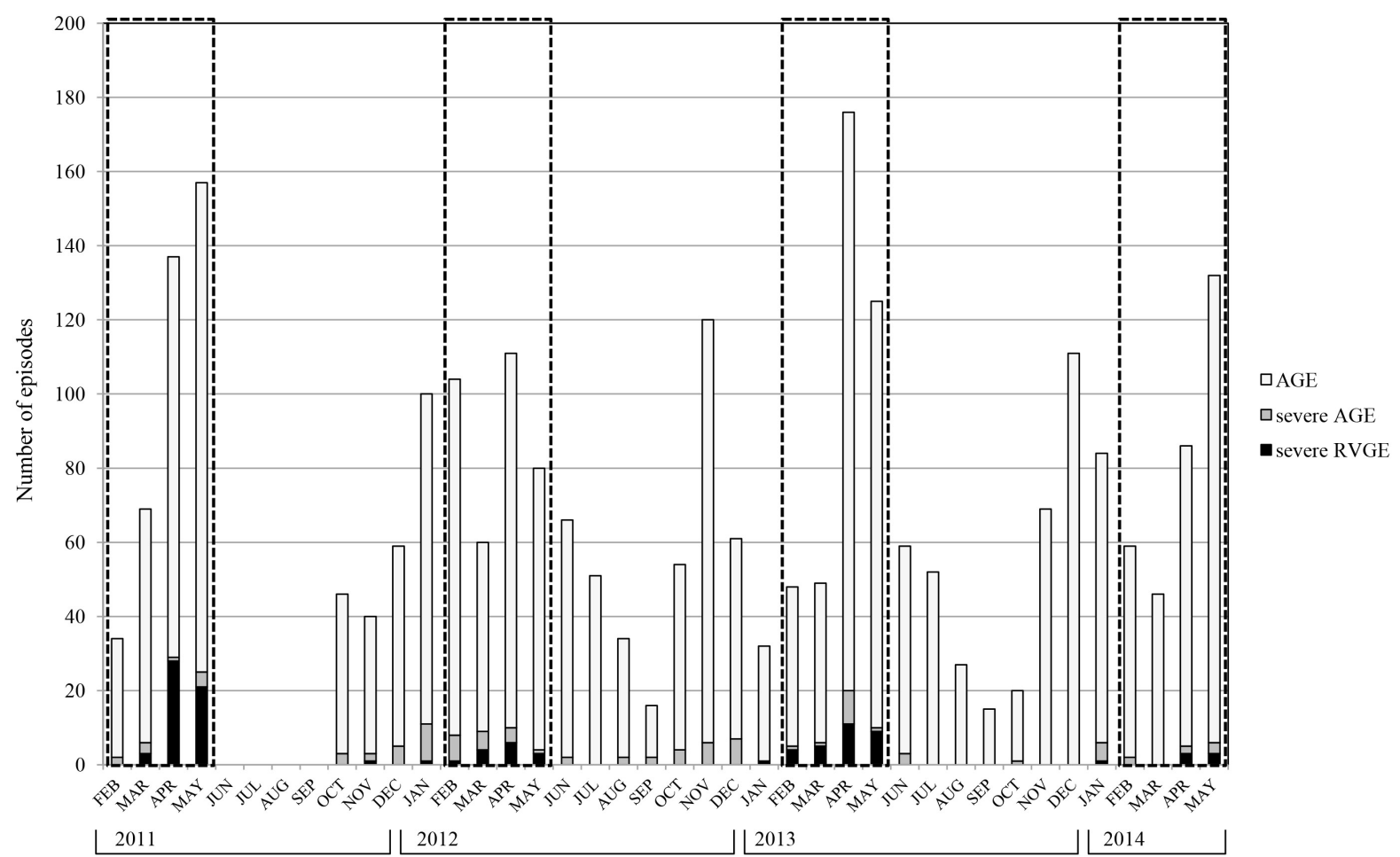

Figure 1. Monthly number of episodes of AGE, severe AGE, and severe RVGE ( $<3$ years of age). Number of AGE (pale gray column), severe AGE (gray column) and severe RVGE (black column) episodes are indicated throughout the study periods. Severe RVGE episodes include only test confirmed episodes. Dotted squares indicate epidemic seasons. In 2011, the study was performed in only the epidemic months from February to May and the months from October to December. The peak month of severe RVGE was April in each year.

Table 3. Occurrence of severe RVGE or severe AGE among all AGE or severe AGE patients.

\begin{tabular}{ccccc}
\hline Occurrence of & $\mathbf{2 0 1 1}$ & $\mathbf{2 0 1 2}$ & $\mathbf{2 0 1 3}$ & $\mathbf{2 0 1 4}$ \\
\hline Severe RVGE/all AGE & $13.1 \%(52 / 397)$ & $4.8 \%{ }^{* *}(17 / 355)$ & $7.8 \%{ }^{*}(31 / 398)$ & $3.4 \%{ }^{* *}(11 / 323)$ \\
Severe RVGE/severe AGE & $83.9 \%(52 / 62)$ & $54.8 \%{ }^{* *}(17 / 31)$ & $75.6 \%(31 / 41)$ & $84.6 \%(11 / 13)$ \\
Severe AGE/all AGE & $15.6 \%(62 / 397)$ & $8.7 \%^{* *}(31 / 355)$ & $10.3 \%{ }^{*}(41 / 398)$ & $4.0 \%{ }^{* *}(13 / 323)$ \\
\hline
\end{tabular}

${ }^{*} \mathrm{p}<0.05 ;{ }^{* *} \mathrm{p}<0.01$ 
2013, and 2014, seasons after RV vaccine introduction, were lower than in 2011, the season before RV vaccine introduction. The occurrences of severe RVGE among severe AGE were similar in each year except for 2012, indicating that the majority of severe GE episodes that require intravenous rehydration in outpatient settings are possibly attributable to rotavirus during RV epidemic months.

\subsection{Changes in Incidence of Severe RVGE}

The incidence rates of severe RVGE were significantly reduced, by $71.2 \%, 47.7 \%$, and $81.1 \%$ for 2012 , 2013, and 2014, respectively, compared to that in 2011, the season before RV vaccine introduction (Table 4). Similar reductions were observed in the incidence rates of severe AGE. Reduction in the incidence rates of AGE were seen in 2012 (21.3\%) and 2014 (27.4\%), but not in 2013.

\subsection{Profiles of Patients with Severe RVGE}

The descriptions of children with severe RVGE are shown in Table 5. The peak months of age were 12 to 23

Table 4. Incidence rates of severe RVGE, severe AGE, and AGE among children younger than 3 years of age.

\begin{tabular}{ccccc}
\hline $\begin{array}{c}\text { /1000 person-years } \\
\text { (95\% CI) }\end{array}$ & $\mathbf{2 0 1 1}$ & $\mathbf{2 0 1 2}$ & $\mathbf{2 0 1 3}$ & $\mathbf{2 0 1 4}$ \\
\hline Severe RVGE & $77.1(57.6-99.5)$ & $22.2(12.9-33.9)^{* * *}$ & $40.3(27.4-55.7)^{* * *}$ & $14.6(7.3-24.4)^{* *}$ \\
\% reduction & - & $71.2 \%(50.3-83.4 \%)$ & $47.7 \%(18.4-66.5 \%)$ & $81.1 \%(63.8-90.1 \%)$ \\
Severe AGE & $92.0(70.5-116.2)$ & $40.5(27.5-55.9)^{* *}$ & $53.3(38.3-70.9)^{* *}$ & $17.2(9.2-27.8)^{* * *}$ \\
\% reduction & - & $56.0 \%(32.3-71.4 \%)$ & $42.1 \%(13.9-60.9 \%)$ & $81.3 \%(66.0-89.7 \%)$ \\
AGE & $588.8(532.3-648.1)$ & $463.4(416.4-512.8)^{* *}$ & $517.8(468.2-569.9)$ & $427.7(382.3-475.6)^{* *}$ \\
\% reduction & - & $21.3 \%(9.2-31.8 \%)$ & $12.1 \%(-1.1-23.5 \%)$ & $27.4 \%(15.9-37.3 \%)$ \\
\hline
\end{tabular}

${ }^{* *} \mathrm{p}<0.01$ vs 2011; Number of children younger than 3 years of age in population as of March 31; 2011: 2300; 2012: $2311 ; 2013: 2338 ; 2014: 2297$. Observation period: 2011: 107 days (Feb 14 to May 31); 2012: 121 days (Feb 1 to May 31); 2013: 120 days (Feb 1 to May 31); $2014: 120$ days (Feb 1 to May 31).

Table 5. Profile of severe RVGE patients.

\begin{tabular}{|c|c|c|c|c|c|}
\hline & & 2011 & 2012 & 2013 & 2014 \\
\hline Total number & 52 & 17 & 31 & 11 & \\
\hline \multirow[t]{2}{*}{ Sex } & Male & 27 & 9 & 16 & 5 \\
\hline & Female & 25 & 8 & 15 & 6 \\
\hline \multirow[t]{4}{*}{ Age in months } & 0 to 5 & 2 & 1 & 0 & 0 \\
\hline & 6 to11 & 7 & 3 & 0 & 1 \\
\hline & 12 to 23 & 24 & 6 & 18 & 2 \\
\hline & 24 to 35 & 19 & 7 & 13 & 8 \\
\hline \multirow[t]{4}{*}{ Months } & February & 0 & 1 & 4 & 1 \\
\hline & March & 3 & 5 & 5 & 0 \\
\hline & April & 28 & 8 & 13 & 5 \\
\hline & May & 21 & 3 & 9 & 5 \\
\hline \multirow[t]{3}{*}{ Vaccinated } & - & 0 & 1 & 2 & \\
\hline & & & & Rotarix $^{\mathrm{TM}} 1$ & Rotarix $^{\mathrm{TM}} 1$ \\
\hline & & & & & RotaTeq $^{\mathrm{TM}} 1$ \\
\hline Severity Score & 11-point Vesikari Scale & $6.04(1.88)$ & $7.00(1.90)$ & $5.77(2.37)$ & $7.00(2.12)$ \\
\hline \multirow[t]{2}{*}{ Mean (SD) } & 20-point Vesikari Scale & - & $12.00(2.90)$ & $11.68(2.10)$ & $10.20(1.64)$ \\
\hline & Number evaluated & 52 & 11 & 22 & 5 \\
\hline
\end{tabular}


months in 2011 and 2013 and 24 to 35 months in 2012 and 2014. The peak month of epidemic season was April. Yearly variation was not detected in the mean severity score calculated using 11-point and 20-point Vesikari's scales [14]. There were 3 severe RVGE episodes observed in children who had received RV vaccine before the onsets: 1 in 2013 and 2 in 2014 (Table 6). All 3 episodes were observed more than one year after vaccination.

\subsection{Genotypes of Rotaviruses}

Genotypes of rotaviruses in feces collected from the study site and Shibata Hospital are summarized in Table 7. The majority genotype was G3P [8] in 2011, G1P [8] and G9P [8] in 2012, G1P [8] in 2013, and G9P [8] in 2014. Epidemic viruses might change year by year.

\subsection{Estimated Vaccine Coverage Rate}

In Shibata City, voluntary RV vaccination started from the end of November 2011. Vaccination costs should have been paid by parents or guardians of infants. From the number of patients who were vaccinated with at least one dose of Rotarix ${ }^{\mathrm{TM}}$ or RotaTeq ${ }^{\mathrm{TM}}$ at the three pediatric clinics and the Shibata Hospital during the study period, vaccine coverage rate in the birth cohort was estimated as $32.5 \%, 40.5 \%$, and $47.1 \%$ as of the end of May in 2012, 2013, and 2014.

\subsection{Changes in RVGE Hospitalization}

The number of children younger than 3 years of age who lived in Shibata City and hospitalized in the Shibata Hospital due to RVGE and AGE was retrospectively collected for reference of long-term trends. Figure 2 indicates numbers of hospitalizations due to RVGE and non-RV GE by year. The proportion of RVGE among AGE was $26.9 \%$ in the period after RV vaccine introduction (from January 2012 to May 2014), significantly lower (p $=0.018$ ) compared with $44.7 \%$ in the period before (from January 2008 to December 2011). Although year-toyear variation was seen in numbers of hospitalizations due to RVGE and AGE, the proportion of RVGE among AGE reduced after $R V$ vaccine introduction.

Table 6. Profiles of severe RVGE patients with vaccination history.

\begin{tabular}{cccc}
\hline & No. 1 & No. 2 & No. 3 \\
\hline Date of diagnosis & $2013 / 2 / 28$ & $2014 / 4 / 28$ & $2014 / 5 / 12$ \\
Age in months & 17 & 25 & 15 \\
Sex & Male & Female & Male $^{\mathrm{TM}}$ \\
Vaccinated with & Rotarix $^{\mathrm{TM}}$ & Rotarix $^{\mathrm{TM}}$ & RotaTeq $^{\mathrm{TM}}$ \\
Vaccinated in & $2012 / 1 ; 2012 / 2$ & $2012 / 6 ; 2012 / 8$ & 11 \\
Severity Score & & Not available $2013 / 5 ; 2013 / 6$ \\
20-point Vesikari Scale & 11 & Not available & 9 \\
10-point Vesikari Scale & 6 & & 2 \\
\hline
\end{tabular}

Table 7. Genotypes of rotaviruses detected during the study period.

\begin{tabular}{|c|c|c|c|c|c|c|c|c|}
\hline & \multicolumn{2}{|c|}{2011} & \multicolumn{2}{|c|}{2012} & \multicolumn{2}{|c|}{2013} & \multicolumn{2}{|c|}{2014} \\
\hline & $n$ & (\%) & $n$ & (\%) & $n$ & $(\%)$ & $n$ & (\%) \\
\hline G1P [8] & 1 & 5.9 & 18 & 45 & 39 & 88.6 & 0 & 0 \\
\hline G2P [4] & 1 & 5.9 & 1 & 2.5 & 5 & 11.4 & 1 & 16.7 \\
\hline G3P [8] & 14 & 82.4 & 1 & 2.5 & 0 & 0 & 0 & 0 \\
\hline G9P [8] & 1 & 5.9 & 20 & 50.0 & 0 & 0 & 5 & 83.3 \\
\hline Total & 17 & & 40 & & 44 & & 6 & \\
\hline
\end{tabular}




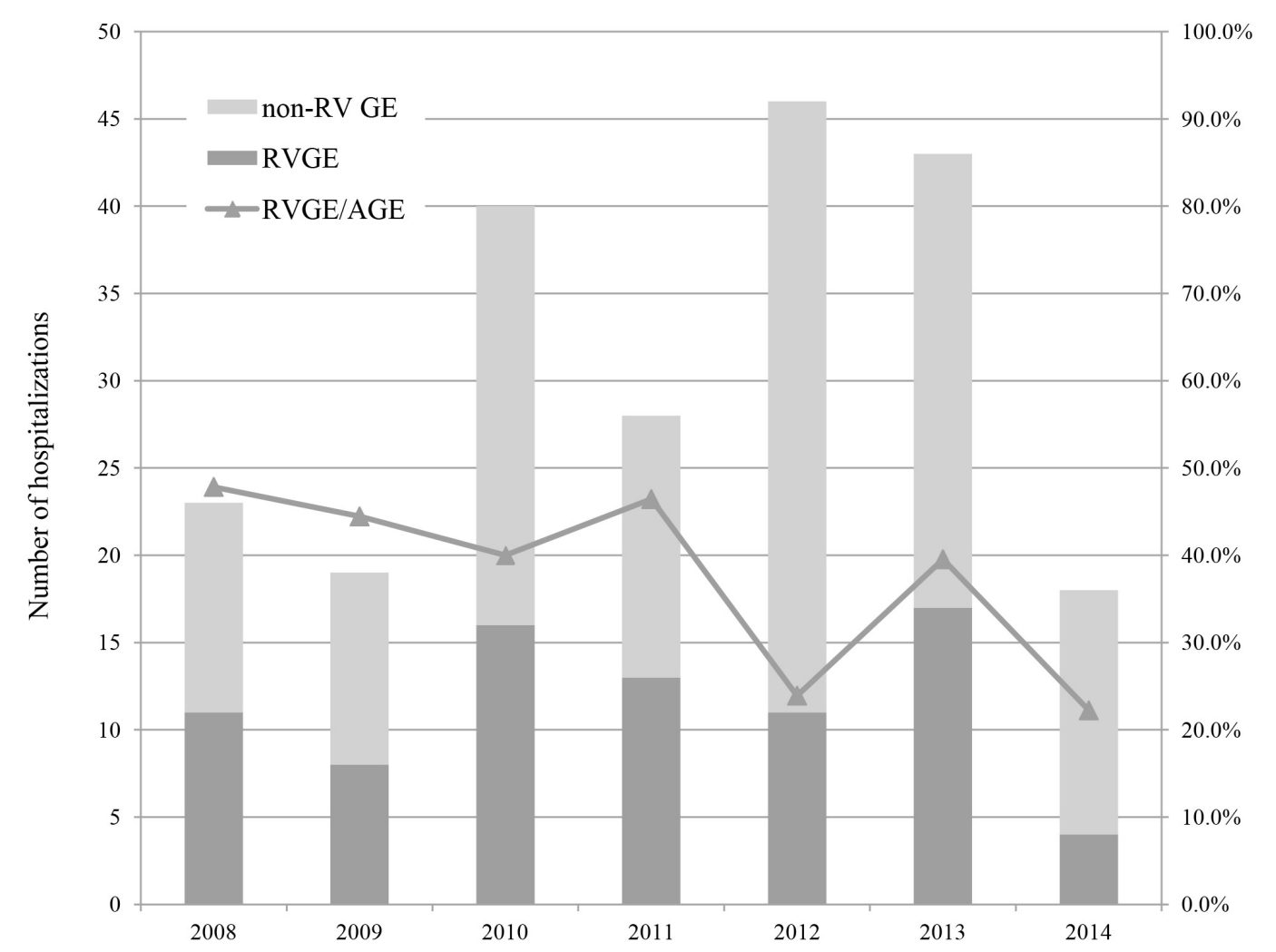

Figure 2. Numbers of RVGE and non-RV GE hospitalizations (children younger than 3 years of age). Numbers of hospitalizations among children younger than 3 years of age who lived in Shibata City are indicated in dark gray column (due to RVGE) and pale gray column (due to non-RV GE) by year. The proportion of RVGE among AGE reduced after the introduction of $\mathrm{RV}$ vaccines compared with the years before. All patients hospitalized due to AGE had RV tests to determine the cause of AGE.

\section{Discussion}

Consistent reduction in the occurrence and incidence rates of severe RVGE in children less than 3 years of age observed in three seasons after RV vaccine introduction may be explained by the vaccine impact. As in many other studies [4]-[6], the results suggest indirect benefits for older children, in addition to the children who were targeted for vaccination. Patel MM et al. described that indirect benefits were noted in the first or the second year after vaccine introduction, while only infants were eligible to receive vaccination, potentially implicating infants as the primary transmitters of infection [6].

In spite of consistent reduction after RV vaccine introduction, relative increases in the incidence rates of AGE, severe AGE, and severe RVGE were seen in 2013, the second season after vaccine introduction. Biennial epidemic peaks after RV vaccine introduction were also observed in some other studies [6] [16]. The biennial pattern was explained by accumulation of unimmunized susceptible children during seasons with low rotavirus activity and the higher number of susceptible children to facilitate transmission during a subsequent season [6] [16].

The occurrence of severe RVGE among severe AGE decreased in 2012 but did not in 2013 and 2014. We consider that a substantial proportion of AGE patients less than 3 years old who require intravenous rehydration therapy are RVGE patients during epidemic months in outpatient settings. The reduction of occurrence in 2012 might be influenced by AGE epidemic of other pathogens such as noro virus.

A major limitation of this study was that we had only one season of observation data as a reference. However, although there was year-to-year variation in numbers of severe RVGE and severe AGE after RV vaccine introduction, consistent reduction in the occurrence and incidence rates of severe RVGE was observed in the three seasons after RV vaccine introduction. It may be reasonable to consider that the reduction is due to $\mathrm{RV}$ vaccine impact. 
Another limitation is that the study was conducted under circumstances in which the vaccine coverage rates were not sufficiently high. There are some studies reporting vaccine impact under relatively low vaccine coverage rates [11] [13] [17]-[20]. Zeller reported 35\% reduction in the RV-positive rate among hospitalized patients aged less than 5 years at a university hospital in Belgium when the vaccine coverage rate was estimated as 65\% [11]. A study in Germany, where the vaccine was introduced in 2006 but not incorporated into the national immunization program as of 2012, evaluated the rates of RVGE hospitalization in the eastern region with high vaccination coverage and in the western region with low vaccination coverage. The results suggested the rates of RVGE hospitalization of infants from 6 to 11 months of age would be reduced by $42 \%$ when the vaccination coverage rates increase to $50 \%[19]$.

In Shibata City, the incidence rates of severe RVGE among outpatients younger than 3 years of age were reduced by $71.2 \%$ in $2012,47.7 \%$ in 2013 , and $81.1 \%$ in 2014. Estimated vaccine coverage rates were $32.5 \%$, $40.5 \%$, and $47.1 \%$ as of the end of May in 2012, 2013, and 2014. There were no direct associations observed between the vaccine coverage rates and the reduction rates of severe RVGE. We assume that the vaccine may be effective for the prevention of severe RVGE even under these moderate vaccination rates in Japan, and that the year-to-year variation may be explained by the biennial epidemic pattern.

\section{Conclusion}

Reductions were observed in the occurrence of severe RVGE among all AGE and in the incidence rates of severe RVGE in three consecutive seasons after introduction of RV vaccine, compared to that in 2011, before introduction of RV vaccines in primary care outpatient settings. These results suggest that RV vaccination may be effective for the prevention of severe RVGE. It is expected that the reducing effect on severe RVGE would be persistently established by increasing the vaccine coverage rates.

\section{Acknowledgements}

This study was supported in part by a research grant from the Investigator-Initiated Studies Program of GlaxoSmithKline Biologicals and Japan Vaccine Co., Ltd.

We would like to thank Dr. Tsutomu Tamura at Niigata Prefectural Institute of Public Health and Environmental Sciences for his cooperation in genotyping of rotavirus. We would also like to thank Satoshi Osaga and Hiromi Sugawara at MC\&P Co., Ltd. for their assistance in statistical analyses and medical writing.

\section{References}

[1] Tate, J.E., Burton, A.H., Boschi-Pinto, C., Steele, A.D., Duque, J. and Parashar, U.D., the WHO-coordinated Global Rotavirus Surveillance Network (2011) 2008 Estimate of Worldwide Rotavirus-Associated Mortalityin Children Younger than 5 Years before the Introduction of Universal Rotavirus Vaccination Programmes: A Systematic Review and Meta-Analysis. The Lancet Infectious Diseases, 12, 136-141. http://dx.doi.org/DOI:10.1016/S1473-3099(11)70253-5

[2] Yokoo, M., Arisawa, K. and Nakagomi, O. (2004) Estimation of Annual Incidence, Age-Specific Incidence Rate, and Cumulative Risk of Rotavirus Gastroenteritis among Children in Japan. Japanese Journal of Infectious Diseases, 57, 166-171.

[3] Nakagomi, T., Nakagomi, O., Takahashi, Y., Enoki, M., Suzuki, T. and Kilgore, P.E. (2005) Incidence and Burden of Rotavirus Gastroenteritis in Japan, as Estimated from a Prospective Sentinel Hospital Study. The Journal of Infectious Diseases, 192, S106-S110. http://dx.doi.org/10.1086/431503

[4] Yen, C., Tate, J.E., Hyde, T.B., Cortese, M.M., Lopman, B.A., Jiang, B., Glass, R.I. and Parashar, U.D. (2014) Rotavirus Vaccines-Current Status and Future Considerations. Human Vaccine \& Immunotherapeutics, 10, 1-13. http://dx.doi.org/10.4161/hv.28857

[5] Patel, M.M., Glass, R., Desai, R., Tate, J.E. and Parashar, U.D. (2012) Fulfilling the Promise of Rotavirus Vaccines: How Far Have We Come Since Licensure? The Lancet Infectious Diseases, 12, 561-570. http://dx.doi.org/10.1016/S1473-3099(12)70029-4

[6] Patel, M.M., Steele, D., Gentsch, J.R., Wecker, J., Glass, R.I. and Parashar, U.D. (2011) Real-World Impact of Rotavirus Vaccination. Pediatric Infectious Disease Journal, 30, S1-S5. http://dx.doi.org/10.1097/INF.0b013e3181fefa1f

[7] World Health Organization (2013) Rotavirus Vaccines WHO Position Paper—January 2013. Weekly Epidemiological record, 88, 49-64. 
[8] Committee for National Immunization Policy (2013) Interim Report of rotavirus Vaccination Working Group (in Japanese). http://www.mhlw.go.jp/file/05-Shingikai-10601000-Daijinkanboukouseikagakuka-Kouseikagakuka/0000029637.pdf

[9] Weinberg, G.A. and Szilagyi, P.G. (2010) Vaccine Epidemiology: Efficacy, Effectiveness, and the Translational Research Roadmap. The Journal of Infectious Diseases, 201, 1607-1610. http://dx.doi.org/10.1086/652404

[10] Clark, H.F., Lawley, D., Mallette, L.A., Dinubile, M.J. and Hodinka, R.L. (2009) Decline in Cases of Rotavirus Gastroenteritis Presenting to The Children's Hospital of Philadelphia after Introduction of a Pentavalent Rotavirus Vaccine. Clinical and Vaccine Immunology, 16, 382-386. http://dx.doi.org/10.1128/CVI.00382-08

[11] Zeller, M., Rahman, M., Heylen, E., De Coster, S., De Vos, S., Arijs, I., Novo, L., Verstappen, N., Van Ranst, M. and Matthijnssens, J. (2010) Rotavirus Incidence and Genotype Distribution before and after National Rotavirus Vaccine Introduction in Belgium. Vaccine, 28, 7507-7513. http://dx.doi.org/10.1016/j.vaccine.2010.09.004

[12] Rae, M., Strens, D., Vergison, A., Verghote, M. and Standaert, B. (2011) Reduction in Pediatric Rotavirus-Related Hospitalizations after Universal Rotavirus Vaccination in Belgium. The Pediatric Infectious Disease Journal, 30, e120e125. http://dx.doi.org/10.1097/INF.0b013e318214b811

[13] Bégué, R.E. and Perrin, K. (2010) Reduction in Gastroenteritis with the Use of Pentavalent Rotavirus Vaccine in a Primary Practice. Pediatrics, 126, e40-e45. http://dx.doi.org/10.1542/peds.2009-2069

[14] Oishi, T., Taguchi, T., Nakano, T., Sudo, S. and Kuwajima, H. (2014) The Occurrence of Severe Rotavirus Gastroenteritis in Children under 3 Years of Age before and after the Introduction of Rotavirus Vaccine: A Prospective Observational Study in Three Pediatric Clinics in Shibata City, Niigata Prefecture, Japan. Japanese Journal of Infectious Diseases, 67, 304-306. http://dx.doi.org/10.7883/yoken.67.304

[15] Ruuska, T. and Vesikari, T. (1990) Rotavirus Disease in Finnish Children: Use of Numerical Scores for Clinical Severity of Diarrhoeal Episodes. Scandinavian Journal of Infectious Diseases, 22, 259-267. http://dx.doi.org/10.3109/00365549009027046

[16] Tate, J.E., Haynes, A., Payne, D.C., Cortese, M.M., Lopman, B.A., Patel, M.M. and Parashar, U.D. (2013) Trends in National Rotavirus Activity before and after Introduction of Rotavirus Vaccine into the National Immunization Program in the United States, 2000 to 2012. The Pediatric Infectious Disease Journal, 32, 741-744. http://dx.doi.org/10.1097/INF.0b013e31828d639c

[17] Curns, A.T., Steiner, C.A., Barrett, M., Hunter, K., Wilson, E. and Parashar, U.D. (2011) Reduction in Acute Gastroenteritis Hospitalizations among US Children after Introduction of Rotavirus Vaccine: Analysis of Hospital Discharge Data from 18 US States. The Journal of Infectious Diseases, 201, 1617-1624. http://dx.doi.org/10.1086/652403

[18] Gil-Prieto, R., Gonzalez-Escaladaa, A., Alvaro-Mecaa, A., Garcia-Garciaa, L., San-Martinb, M., González-Lópezb, A. and Gil-de-Miguela, A. (2013) Impact of Non-Routine Vaccination on Hospitalizations for Diarrhoea and Rotavirus Infections in Spain. Vaccine, 31, 5000-5004. http://dx.doi.org/10.1016/j.vaccine.2013.05.109

[19] Dudareva-Vizule, S., Koch, J., an der Heiden, M., Oberle, D., Keller-Stanislawski, B. and Wichmann, O. (2012) Impact of Rotavirus Vaccination in Regions with Low and Moderate Vaccine Uptake in Germany. Human Vaccine \& Immunotherapy, 8, 1407-1415. http://dx.doi.org/10.4161/hv.21593

[20] Steyer, A., Sagadin, M., Kolenc, M. and Poljšak-Prijatelj, M. (2014) Molecular Characterization of Rotavirus Strains from Pre- and Post-Vaccination Periods in a Country with Low Vaccination Coverage: The Case of Slovenia. Infection, Genetics and Evolution, in press. http://dx.doi.org/10.1016/j.meegid.2014.06.021 
Scientific Research Publishing (SCIRP) is one of the largest Open Access journal publishers. It is currently publishing more than 200 open access, online, peer-reviewed journals covering a wide range of academic disciplines. SCIRP serves the worldwide academic communities and contributes to the progress and application of science with its publication.

Other selected journals from SCIRP are listed as below. Submit your manuscript to us via either submit@scirp.org or Online Submission Portal.
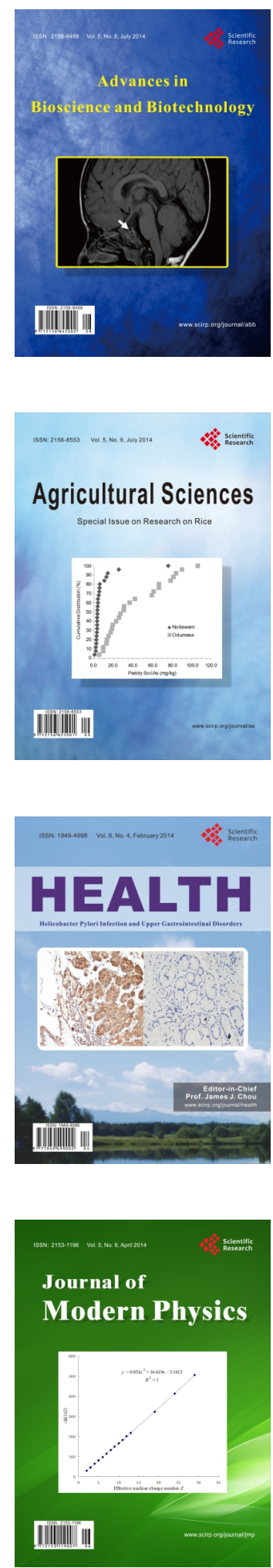
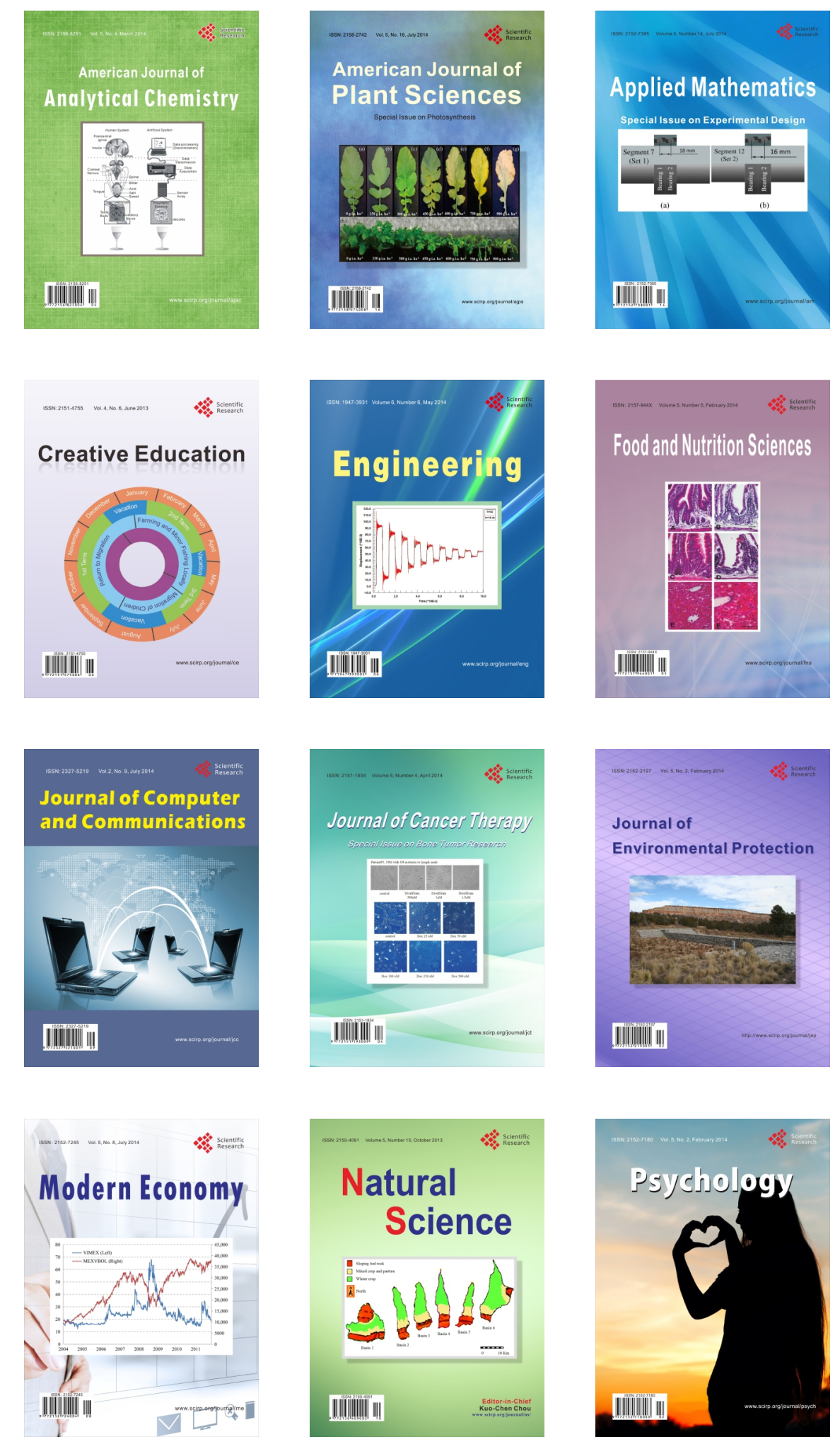